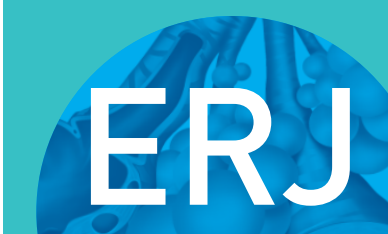

open research
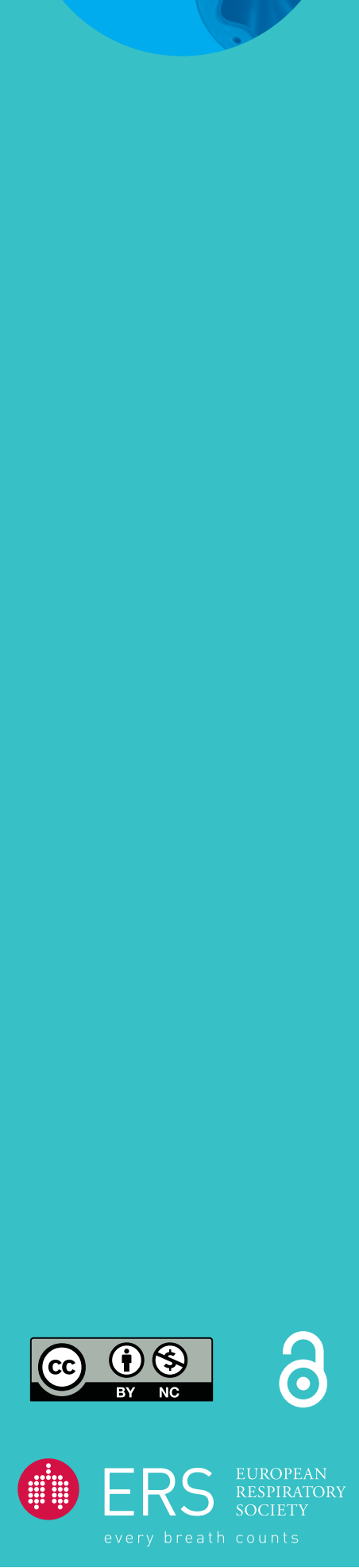

\section{Lung clearance index in detection of post-transplant bronchiolitis obliterans syndrome}

\author{
Madeleine Driskel (10 ${ }^{1,2}$, Alex Horsley $\mathbb{1}^{3}{ }^{3}$, Laurice Fretwell ${ }^{4}$, Nigel Clayton ${ }^{1}$ and \\ Mohamed Al-Aloul ${ }^{2}$
}

\begin{abstract}
Affiliations: '1Lung Function Laboratory, Manchester University NHS Foundation Trust, Manchester, UK ${ }^{2}$ Cardiothoracic Transplant Unit, Manchester University NHS Foundation Trust, Manchester, UK. ${ }^{3}$ Faculty of Biology, Medicine and Health, University of Manchester, Manchester, UK. ${ }^{4}$ School of Human Sciences, University of Derby, Derby, UK.
\end{abstract}

Correspondence: Alex Horsley, Division of Infection, Immunity and Respiratory Medicine, Faculty of Biology, Medicine and Health, University of Manchester, Manchester M23 9LT, UK.

E-mail: alexander.horsleyamanchester.ac.uk

\section{ABSTRACT}

Background: Long-term outcomes after lung transplantation are often limited by the development of obliterative bronchiolitis (OB), which is clinically defined using spirometry as bronchiolitis obliterans syndrome (BOS). Lung clearance index (LCI), derived from multiple breath washout (MBW) testing, is a global measure of ventilation heterogeneity that has previously been shown to be a more sensitive measure of obstructive small airway diseases than spirometry. We aimed to assess the feasibility of LCI in adult lung transplant patients and to compare LCI to BOS grade.

Methods: 51 stable adult double-lung transplant recipients performed sulfur hexafluoride MBW in triplicate on a single occasion, using a closed-circuit Innocor device. BOS grades were derived from serial spirometry according to International Society for Heart and Lung Transplantation criteria and, where available, high-resolution computed tomography (HRCT) evidence of OB was recorded.

Results: LCI was successfully performed in $98 \%$ of patients. The within-visit coefficient of variation for repeat LCI measurements was $3.1 \%$. Mean LCI increased significantly with BOS grades: no BOS $(n=15)$, LCI 7.6; BOS-0p ( $n=16)$, LCI 8.3; BOS-1 ( $\mathrm{n}=11)$, LCI 9.3; BOS-2-3 $(\mathrm{n}=9)$, LCI $13.2(\mathrm{p}<0.001) .27$ patients had HRCT within 12 months. LCI in those with HRCT evidence of OB was higher than those without OB (11.1 versus $8.2, \mathrm{p}=0.006)$. $47 \%$ patients displayed abnormal LCI $(>7)$ despite a normal forced expiratory volume in $1 \mathrm{~s}\left(\mathrm{FEV}_{1}\right)(>80 \%$ of baseline).

Conclusions: LCI measurement in lung transplant recipients is feasible and reproducible. LCI increased with increasing BOS grade. A significant proportion of this cohort had abnormal LCI with preserved $\mathrm{FEV}_{1}$, suggesting early subclinical small airway dysfunction, and supporting a role for $\mathrm{MBW}$ in the early identification of BOS.

@ERSpublications

BOS limits post-lung transplant outcomes. LCI and phase III slope indices, derived from MBW measured by a novel closed-circuit technique, increase with BOS severity and may allow detection of mild disease before conventional spirometric methods. http://bit.ly/2L113yC

Cite this article as: Driskel M, Horsley A, Fretwell L, et al. Lung clearance index in detection of post-transplant bronchiolitis obliterans syndrome. ERJ Open Res 2019; 5: 00164-2019 [https:// doi.org/10.1183/23120541.00164-2019].

This article has supplementary material available from openres.ersjournals.com

Received: 16 July 2019 | Accepted after revision: 16 Aug 2019

Copyright $\odot E R S$ 2019. This article is open access and distributed under the terms of the Creative Commons Attribution Non-Commercial Licence 4.0. 


\section{Introduction}

Chronic lung allograft dysfunction (CLAD) curtails medium- and long-term outcomes after lung transplantation [1]. Obliterative bronchiolitis (OB) is the predominant phenotype of CLAD and is typified by lymphocytic infiltration of the bronchioles, peribronchiolar fibrosis and constriction of the bronchial lumen [2]. This pathology is not uniform throughout the graft and is seldom captured by surveillance transbronchial biopsies, making histological confirmation of $\mathrm{OB}$ challenging. The term bronchiolitis obliterans syndrome (BOS) has therefore been adopted as a clinical surrogate for $O B$. This reflects progressive airflow limitation diagnosed by serial spirometry testing during routine clinical follow-up. The International Society for Heart and Lung Transplantation (ISHLT) defines BOS as a $\geqslant 20 \%$ drop in forced expiratory volume in the first second $\left(\mathrm{FEV}_{1}\right)$ from a stable post-transplant baseline, persistent over $\geqslant 3$ weeks and not explained by other reversible pathologies [1]. Air trapping on expiratory high-resolution computed tomography (HRCT) of the chest further supports the diagnosis, whilst excluding alternative causes of $\mathrm{FEV}_{1}$ decline. Spirometry, however, is recognised to be poor at detecting early small airway changes [3]. Considerable peripheral lung damage can occur in airways $<2 \mathrm{~mm}$ in diameter before this is sufficient to impact on air flows at the mouth $[4,5]$.

Multiple breath washout (MBW) testing is an alternative assessment of lung physiology that measures efficiency of gas mixing in the lungs by following the washout of an inert tracer gas during normal relaxed breathing [3]. In the presence of airway disease, there is uneven filling and emptying of different lung regions. This reduces efficiency of tracer gas washout and increases the lung clearance index (LCI), a global measure of ventilation heterogeneity [6]. LCI is very sensitive to early airways disease [7] and has been widely adopted by the cystic fibrosis (CF) community in clinical studies and trials [8], as well as being used in asthma and COPD [9, 10]. In a past study of lung transplant patients, ventilation heterogeneity appeared to be an early feature of BOS [11]. Recent assessments of MBW have all supported this, despite being predominantly small cross-sectional studies in differing populations, and using different tracer gases and indices of ventilation heterogeneity [12-14].

MBW testing is not without its challenges, however, including in particular the time taken to complete testing, which can be prolonged even in those with relatively mild disease [10]. Clinical scalability requires a test that is both sensitive to $\mathrm{OB}$ and clinically practical. Recent advances in MBW methodology offers the possibility of shorter test times [15] but has not yet been widely reported in practice [16]. We hypothesised that MBW would be feasible and reproducible in adult lung transplant recipients, and that LCI would be able to differentiate between patients with different BOS grades. The aims of this study were therefore:

- to determine feasibility of LCI testing using a closed-circuit wash-in method in an outpatient clinical setting in adult lung transplant patients, defined as measurement success rates, testing time and reproducibility of repeat measurements;

- to compare LCI to spirometry; and

- to compare LCI to ISHLT BOS grades (defined spirometrically) and to HRCT evidence of OB.

\section{Patients and methods \\ Clinical setting}

This was a cross-sectional study of clinically stable, adult, double-lung (DLTx) or heart-lung transplant (HLTx) recipients, $\geqslant 18$ years of age, attending routine review at the Manchester Cardiothoracic Unit (Manchester, UK), running recruitment and data collection between May 2017 and 2018. Patients were recruited sequentially from the clinic depending on the availability of staff. The study size was opportunistic, and patients were excluded if clinically unstable, undergoing active treatment for acute pulmonary and/or extrapulmonary complications, colonised with communicable infections or unable to perform reproducible spirometry. Patients gave informed consent and study procedures were carried out in accordance with good clinical practice guidelines and the Declaration of Helsinki. This study was approved by the local Research Ethics Committee (ref. 13/NW/0248).

\section{Lung function measurements}

More detailed descriptions of each of the assessments can be found in the supplementary material. Briefly, MBW was performed using sulfur hexafluoride $\left(\mathrm{SF}_{6}\right)$ as the inert tracer gas, measured using an Innocor photoacoustic gas analyser (Innovision, Odense, Denmark) and a closed-circuit wash-in as previously described [15]. At least three wash-out measurements were conducted and reported outcomes are the mean of measures from at least two successful repetitions. LCI was defined as the number of lung turnovers needed to wash $\mathrm{SF}_{6}$ to $<1 / 40$ th of the starting concentration. The upper limit of normal for LCI was 7 (supplementary material) [17]. 
Test time was derived from the wash-out file length (in seconds, and presented in minutes). This includes device warm-up time ( $60 \mathrm{~s}$ per test) and any intervals between tests. It does not include time taken to explain the test or clean the apparatus between patients.

Routine spirometry was performed after MBW using a VMAX 22 system (SensorMedics, Yorba Linda, CA, USA) in accordance with American Thoracic Society/European Respiratory Society guidelines [18]. Results are expressed as percentage of baseline, i.e. best value achieved post-transplant [1]. All tests were carried out by a single physiologist (M. Driskel)

\section{BOS status}

The BOS stage for each patient was calculated according to ISHLT spirometric thresholds. No BOS was defined as $\mathrm{FEV}_{1}>90 \%$ of baseline and forced expiratory flow at $25-75 \%$ of forced vital capacity $\left(\mathrm{FEF}_{25-75 \%}\right)>75 \%$ of baseline, BOS-0p (pre-BOS) as $\mathrm{FEV}_{1} 81-90 \%$ of baseline and/or $\mathrm{FEF}_{25-75 \%} \leqslant 75 \%$ of baseline, BOS-1 as FEV 1 66-80\%, BOS-2 as $\mathrm{FEV}_{1}$ 51-65\%, and BOS 3 as $\mathrm{FEV}_{1} \leqslant 50 \%$ [1].

For HRCT diagnosis of OB, expiratory HRCT examinations within 12 months of the study visit were included and expiratory air trapping explicitly attributed to OB by the reporting radiologist was noted.

\section{Statistical analysis}

All data were analysed using Prism version 6.04 (GraphPad Software Inc., La Jolla, CA, USA). The D'Agostino and Pearson normality test was used to determine data distribution. The Kruskal-Wallis test was used to compare LCI across BOS stages. Patients with BOS grades 2 and 3 (comprising seven and two patients respectively) were grouped for analysis. For this exploratory dataset, the primary comparisons of clinical interest were differences in LCI between patients with no BOS and those with BOS-0p and BOS-1, as well as between those with BOS-0p and BOS-1. Therefore, to retain the power of the study to detect these clinically relevant differences we have not made adjustments for multiple comparisons and present all paired data with unadjusted $\mathrm{p}$-values, with significance set at $\mathrm{p}<0.05$. For post hoc analysis, group means were compared with unpaired Student's t-test for normally distributed data and presented as mean \pm SD. Mann-Whitney U-tests were used for nonparametric data and quoted as median (interquartile range (IQR)).

\section{Results}

\section{Patient cohort}

Table 1 summarises the clinical characteristics and demographics of the study cohort, comprising 49 DLTx and two HLTx recipients. COPD was the main indication for lung transplantation $(n=24)$, with CF, pulmonary hypertension and interstitial lung disease accounting for 15, seven and five, respectively. Median (IQR) age was 54 (43-63) years, with a median time since transplantation of 4 (0.9-5.9) years.

All patients received induction immunosuppression with antithymocyte globulin, and were maintained on a combination of calcineurin inhibitors, cell cycle inhibitors and prednisolone. None was receiving mTOR inhibitors during the study.

\section{Practicality of MBW in lung transplant}

55 patients attempted MBW testing, which was completed in 54 (98\%) (supplementary material). One subject was excluded from analysis due to inability to complete the test reliably; this patient was also unable to perform technically acceptable spirometry results. Three patients' datasets were also excluded due to a technical fault in the flowmeter, which was subsequently rectified. The final study cohort was therefore 51 patients.

Median (IQR) testing duration was 24 (15-39) min. The median (IQR) coefficient of variation for LCI and functional residual capacity was $3.1(2.1-5.2)$ and $3.4(2.3-4.9)$ respectively.

\section{Comparison of $\mathrm{LCl}$ with spirometry}

43 (84\%) patients had an elevated LCI. Figure 1 shows LCI plotted against $\mathrm{FEV}_{1}$ and $\mathrm{FEF}_{25-75 \%}$ (both expressed as \% of baseline). 24 (47\%) out of 51 patients had an abnormal LCI but a preserved $\mathrm{FEV}_{1}$ (>80\%), whilst $10(20 \%)$ patients had an abnormal LCI with both normal $\mathrm{FEV}_{1}$ and $\mathrm{FEF}_{25-75 \% \text {. }}$

\section{LCl across ISHLT BOS staging}

LCI measurements differed significantly by BOS stage ( $\mathrm{p}<0.0001$, Kruskal-Wallis test) (figure 2 and table 1). In those with BOS- $0 \mathrm{p}$ (pre-BOS), $88 \%$ had an abnormal LCI, with a mean \pm SD of $8.3 \pm 1.0$ compared with $7.6 \pm 0.84$ in those without BOS $(\mathrm{p}=0.0300)$. A significant difference was also observed between those without BOS and BOS-1 $(\mathrm{p}<0.0001)$, as well as those with BOS-0p and BOS-1 $(\mathrm{p}=0.027$, all analysed with 
TABLE 1 Summary of demographics and results across International Society for Heart and Lung Transplantation bronchiolitis obliterans syndrome (BOS) grades

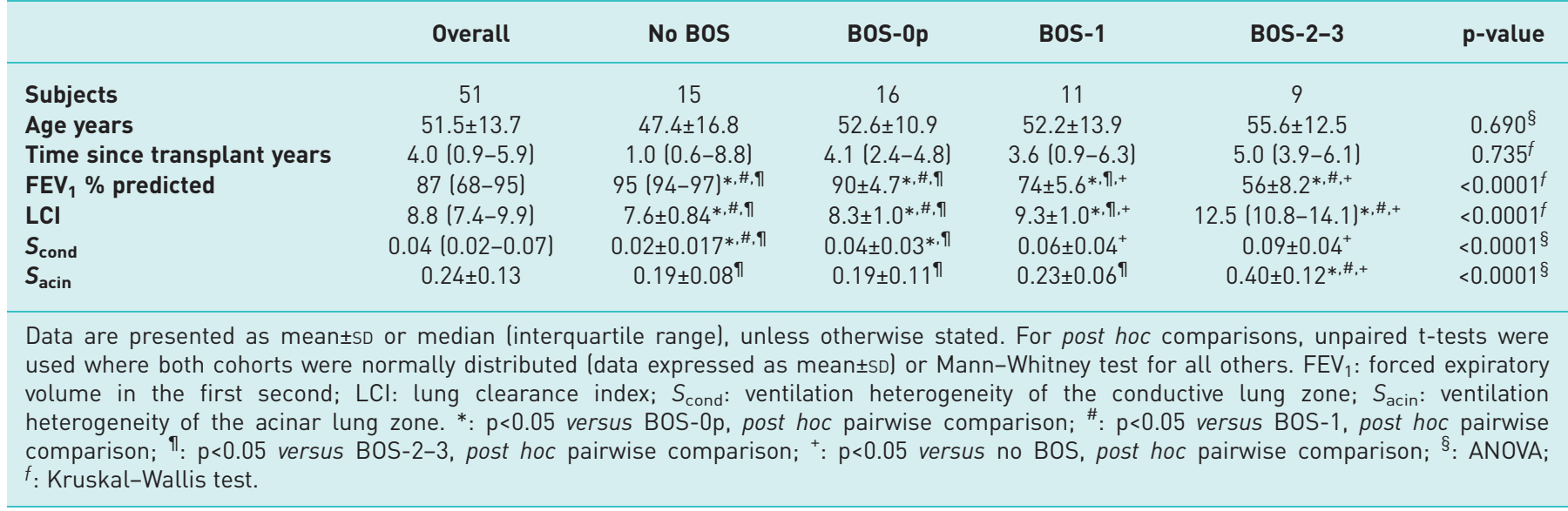

unpaired t-test). BOS-2-3 was significantly higher than all other stages in pairwise comparisons (Mann-Whitney test).

\section{$\mathrm{LCl}$ versus $H R C T$}

27 patients had HRCT results available within 12 months of MBW testing. LCI was significantly higher in those with HRCT evidence of BOS $(n=17)$ compared to those with no BOS on HRCT $(n=10)$ : median (IQR) LCI 10.1 (9.1-12.9) versus 8.2 (7.1-9.3) ( $\mathrm{p}=0.005$, Mann-Whitney) (figure 3). In the 24 patients who did not have recent HRCT, median (IQR) LCI was 8.1 (7.2-8.8).

\section{Cardiogenic oscillations}

In 27 (53\%) patients, potentially significant cardiogenic oscillations were observed during wash-out. These were graded as mild, moderate or severe based on potential impact on wash-out analysis (supplementary material). In eight patients, these oscillations were severe enough to affect phase 3 slope analysis, as an accurate phase 3 slope could not be identified throughout the traces. In seven washouts, this also meant a clear end-point for wash-out could not be unequivocally determined due to superimposed oscillations. Exclusion of these data did not affect the pattern of results across ISHLT BOS grades.

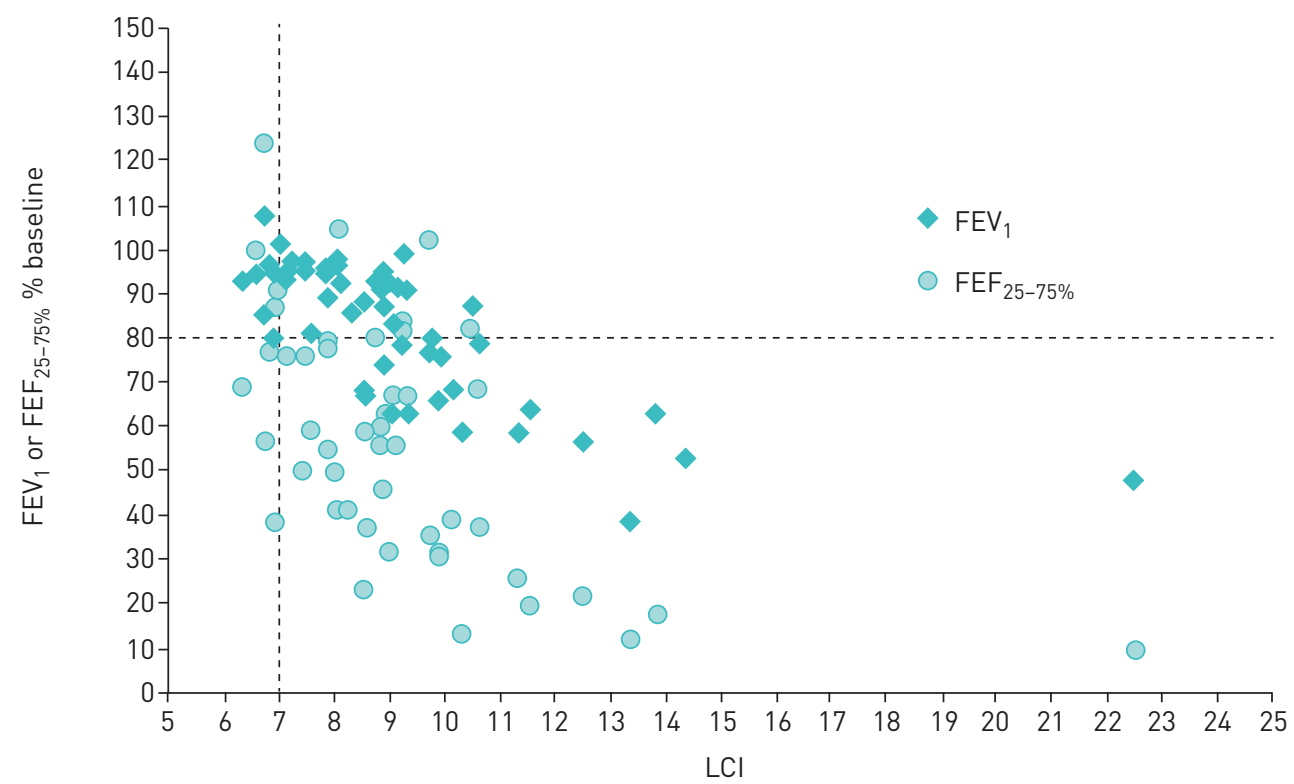

FIGURE 1 Forced expiratory volume in the first second $\left(\mathrm{FEV}_{1}\right)$ and forced expiratory flow at $25-75 \%$ of forced vital capacity $\left(\mathrm{FEF}_{25-75 \%}\right)$ values as \% best from baseline, against lung clearance index (LCI). 
FIGURE 2 Lung clearance index (LCI) against International Society for Heart and Lung Transplantation (ISHLT) bronchiolitis obliterans syndrome (BOS) grade. Bars represent group mean and standard deviation. $p$-values represent unpaired t-tests. Median LCl for BOS-2-3 was statistically higher than in all other groups $(p<0.001$, Mann-Whitney test).

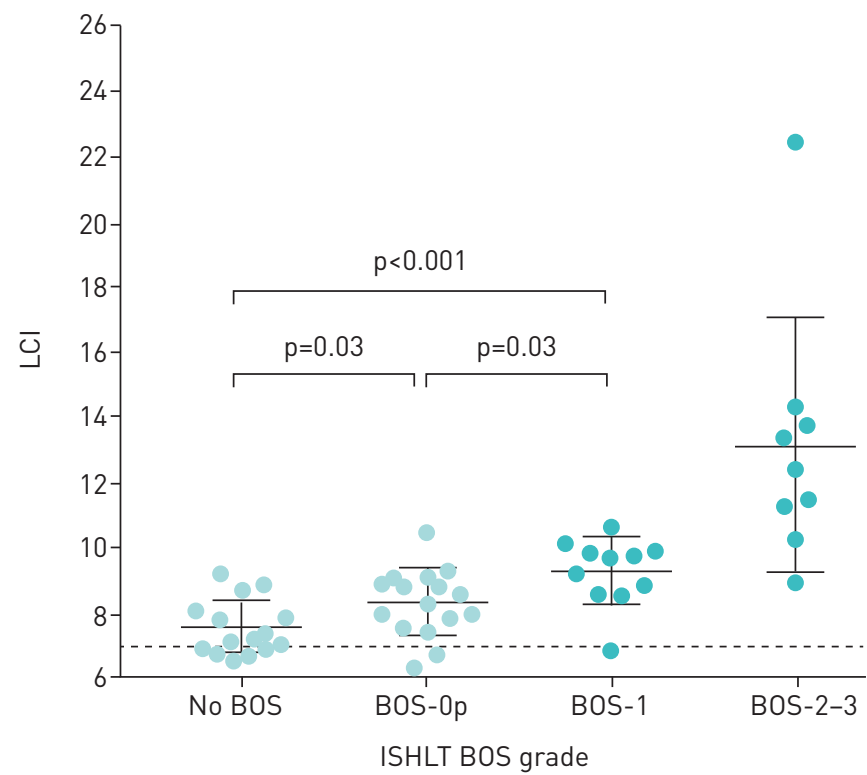

\section{Phase 3 slope indices}

Phase 3 slope analysis was successfully performed in $43(84 \%)$ patients. $S_{\text {acin }}$ was seen to increase along with BOS grade and measures differed significantly between the four groups $(p<0.0001$, one-way ANOVA) (table 1 and figure 4). No significant difference was observed in pairwise comparisons between the milder stages (no BOS to BOS-1, t-test). Ventilation heterogeneity of the conductive lung zone $\left(S_{\text {cond }}\right)$ also increased with BOS severity $(\mathrm{p}<0.0001$, one-way ANOVA). Amongst the milder stages, significant differences were seen between those with no BOS and BOS-0p $(p=0.030)$, and no BOS and BOS-1 ( $\mathrm{p}=0.002$, both $\mathrm{t}$-test $)$.

\section{Discussion}

This is the first study to report several MBW indices in a sizeable cohort of adult lung transplant recipients and to stratify results by BOS grade rather than simply the presence or absence of BOS. We have shown that LCI and $S_{\text {cond }}$ increase with increasing BOS grade and separately, that LCI is higher in those with HRCT evidence of OB. Importantly, we have also shown that LCI is feasible in an outpatient setting using a novel rapid wash-in method, and that this allows measurements to be completed in $<30$ mins with high success rates and within-visit repeatability. Measurement times and repeatability are as good as or

FIGURE 3 Lung clearance index (LCI) in patients with bronchiolitis obliterans syndrome (BOS) on highresolution computed tomography (HRCT), no BOS on HRCT and those with no HRCT performed. Bars represent group mean and standard deviation. \#: Mann-Whitney test; ๆ: t-test.

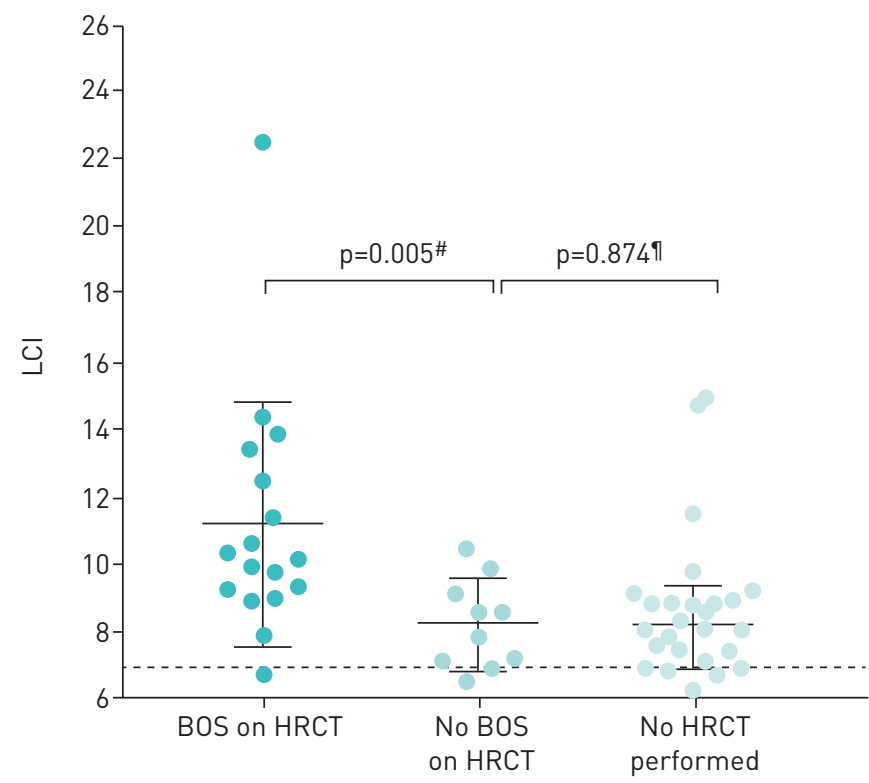



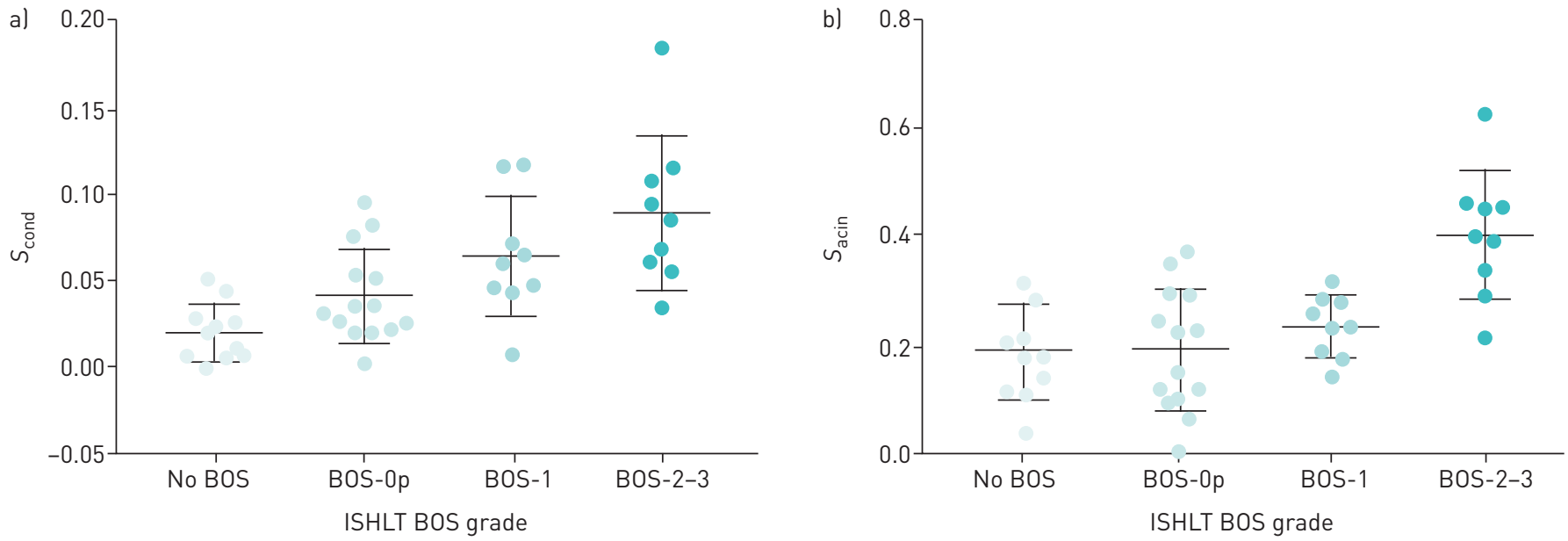

FIGURE 4 a) Ventilation heterogeneity of the conductive lung zone $\left(S_{\text {cond }}\right)$ and b) ventilation heterogeneity of the acinar lung zone $\left(S_{\text {acin }}\right)$ against International Society for Heart and Lung Transplantation (ISHLT) bronchiolitis obliterans syndrome (BOS) grade. Bars represent group mean and standard deviation. For details of the statistical differences between groups, see table 1.

better than those reported for LCI in other populations $[10,19,20]$. These are important initial steps in establishing LCI as an outcome measure for post-transplant monitoring of OB.

ESTENNE et al. [11] have previously shown that single-breath wash-out tests became abnormal in post-lung transplant monitoring an average of 18 months before changes were seen in spirometry. Technology and understanding have progressed substantially since this initial work, and LCI has now become well established in CF clinical and research practice [21]. LCI is very sensitive to early CF changes [19, 22], progresses in the face of unchanged spirometry [23], responds better to interventions [24], and correlates better with radiographic evidence of structural disease and air trapping than spirometry [25]. In contrast, only a small number of MBW studies have been performed in populations with $\mathrm{OB}[13,14,26]$. These have all differed considerably in the technologies and tracer gases used to perform MBW, as well as the populations being studied (including $\mathrm{OB}$ due to the effects of chronic rejection and inflammation in the donor lungs post-transplant and due to graft versus host disease in bone marrow transplant recipients). The cumulative evidence would therefore indicate that ventilation heterogeneity is an early feature of OB, irrespective of its pathogenesis. These studies all lend support to the hypothesis that assessments of ventilation heterogeneity are able to detect OB before it is sufficiently advanced or widespread to reduce airflow at the mouth, as captured by spirometry. We have also shown here that LCI is scalable and that it is elevated in those with pre-BOS, a putative early stage of OB that may be more reversible and amenable to future therapeutic intervention. These findings are supported by a recent study on ventilation magnetic resonance imaging (MRI) in paediatric stem-cell transplant patients, which showed evidence of ventilation defects even in those with normal spirometry [27]

Since spirometry is used to diagnose BOS, we do not have an independent gold standard against which to compare sensitivity of LCI and spirometry. However, only a single patient had a normal LCI but a low $\mathrm{FEV}_{1}$, supporting the hypothesis that LCI is more sensitive than $\mathrm{FEV}_{1}$ to early $\mathrm{OB}$. In addition, $\mathrm{FEF}_{25-75 \%}$, which is believed to capture small airway mechanics more closely than $\mathrm{FEV}_{1}$, displayed better concordance with LCI than did $\mathrm{FEV}_{1}$ (75\% versus 47\%). Without detailed history of the donor lungs, it is possible that elevated LCI may in some cases reflect the pre-transplant history of the lungs rather than recent OB. However, there is good separation based on LCI between patients with different grades of BOS, and also between those with and without independent evidence of OB from HRCT.

Phase 3 slope analysis has been used in previous studies to separate gas mixing abnormalities into components based on gas flow in the airways $\left(S_{\text {cond }}\right)$ and gas diffusion-convection interactions in the lung periphery (ventilation heterogeneity of the acinar lung zone $\left(S_{\text {acin }}\right)$ ). In our study, LCI appeared to perform best at separating the different BOS grades, followed by $S_{\text {cond }}$ and then $S_{\text {acin }}$. $S_{\text {cond }}$, but not $S_{\text {acin }}$, separated BOS-0 from the milder stages of BOS (BOS-0p and BOS-1). Conversely, THOMPSON et al. [28] showed an increase in $S_{\text {acin }}$ but not $S_{\text {cond }}$ across BOS stages using nitrogen washout, although in their study, the only statistically significant differences were between $S_{\text {acin }}$ in established BOS stages 1-3 versus 0 , but not BOS-0p. There are important differences between the two study methods however, in particular the choice of tracer gas. Our data would suggest that it is only when disease in the conductive compartment reaches a certain threshold, represented by the higher grades of BOS, that abnormalities in peripheral gas mixing 
become evident from phase 3 slope analysis. Use of dynamic functional and structural imaging such as ventilation MRI may help inform this debate in future.

One new observation from this study was the presence of very significant cardiogenic oscillations superimposed on the gas wash-out traces in a proportion of wash-outs. These are a well-recognised phenomenon in MBW, first described in 1954 and attributed to heart-synchronous fluctuations in gas concentration, flow or pressure within the lungs [29]. There are no previous detailed reports of the incidence or severity of cardiogenic oscillations, but these wash-out traces were analysed by an experienced team and they are rarely encountered to the degree seen here, either in health or disease. The high incidence of this phenomenon may be a consequence of lung transplant surgery, which involves opening the pericardium and sewing the donor left atrial cuff onto the recipient left atrium. This will conceivably alter cardiopulmonary haemodynamics and pave the way for more prominent cardiogenic oscillations [30].

The main limitation of this study is the cross-sectional design. Thus, although we are able to report on LCI post-transplant, it is not possible to know how much of the abnormality seen is due to disease progression. These are details that can only be explored in a longitudinal study. A strength of this study is that we have used an exogenous tracer gas $\left(\mathrm{SF}_{6}\right)$ to perform $\mathrm{MBW}$, and a closed-circuit wash-in that has allowed more rapid completion of the assessments than the conventional open-circuit method.

In summary, we have shown that MBW is feasible and reproducible in a population of ambulant, adult DLTx or HLTx recipients. Our novel closed-circuit method and portable apparatus allow more rapid testing and increase clinical applicability. These data also demonstrate the potential for increased sensitivity of LCI in the early and more mild stages of BOS evolution.

Acknowledgements: The authors would like to thank Gary Nolan of the lung function laboratory at Wythenshawe Hospital (Manchester, UK) and all the patients who took part in this study.

Conflict of interest: M. Driskel has nothing to disclose. A. Horsley reports grants from the National Institute for Health Research during the conduct of the study. L. Fretwell has nothing to disclose. N. Clayton has nothing to disclose. M. Al-Aloul has nothing to disclose.

Support statement: This work was co-funded by New Start, the heart and lung transplant charity based in Manchester (grant Alaloul 2017), and supported by the National Institute for Health Research (NIHR) Manchester Biomedical Research Centre. This work was carried out at the NIHR Manchester Clinical Research Facility. A. Horsley was supported by an NIHR clinician scientist award (NIHRCS012-13). This report therefore represents independent research funded by the NIHR. The views expressed are those of the authors and not necessarily those of the UK National Health Service, the NIHR or the UK Dept of Health. Funding information for this article has been deposited with the Crossref Funder Registry.

\section{References}

1 Chambers DC, Yusen RD, Goldfarb SB, et al. Registry of the International Society for Heart and Lung Transplantation: twentieth Pediatric Lung and Heart-Lung Transplantation report - 2017; focus theme: allograft ischemic time. J Hear Lung Transplant 2017; 36: 1070-1079.

2 Meyer KC, Raghu G, Verleden GM, et al. An international ISHLT/ATS/ERS clinical practice guideline: diagnosis and management of bronchiolitis obliterans syndrome. Eur Respir J 2014; 44: 1479-1503.

3 Horsley A, Siddiqui S. Putting lung function and physiology into perspective: cystic fibrosis in adults. Respirology 2015; 20: 33-45.

4 Brown R, Woolcock AJ, Vincent NJ, et al. Physiological effects of experimental airway obstruction with beads. J Appl Physiol 1969; 27: 328-335.

5 Brody AS, Klein JS, Molina PL, et al. High-resolution computed tomography in young patients with cystic fibrosis: distribution of abnormalities and correlation with pulmonary function tests. J Pediatr 2004; 145: 32-38.

6 Horsley A. Lung clearance index in the assessment of airways disease. Respir Med 2009; 103: 793-799.

7 Marshall H, Horsley A, Taylor C, et al. Detection of early subclinical lung disease in children with cystic fibrosis by lung ventilation imaging with hyperpolarised gas MRI. Thorax 2017; 72: 760-762.

8 Kent L, Reix P, Innes JA, et al. Lung clearance index: evidence for use in clinical trials in cystic fibrosis. J Cyst Fibros 2014; 13: 123-138.

9 Kjellberg S, Houltz B, Zetterstrom O, et al. Clinical characteristics of adult asthma associated with small airway dysfunction. Respir Med 2016; 117: 92-102.

10 Bell AS, Lawrence PJ, Singh D, et al. Feasibility and challenges of using multiple breath washout in COPD. Int J Chron Obstruct Pulmon Dis 2018; 13: 2113-2119.

11 Estenne M, Van Muylem A, Knoop C, et al. Detection of obliterative bronchiolitis after lung transplantation by indexes of ventilation distribution. Am J Respir Crit Care Med 2000; 162: 1047-1051.

12 Thompson BR, Hodgson YM, Kotsimbos T, et al. Bronchiolitis obliterans syndrome leads to a functional deterioration of the acinus post lung transplant. Thorax 2014; 69: 487-488.

13 Nyilas S, Baumeler L, Tamm M, et al. Inert gas washout in bronchiolitis obliterans following hematopoietic cell transplantation. Chest 2018; 154: 157-168.

14 Kao JE, Zirbes JM, Conrad CK, et al. Lung clearance index is sensitive to small airway disease in pediatric lung transplant recipients. J Heart Lung Transplant 2017; 36: 980-984.

15 Horsley AR, O'Neill K, Downey DG, et al. Closed circuit rebreathing to achieve inert gas wash-in for multiple breath wash-out. ERJ Open Res 2016; 2: 00042-2015. 
Trinkmann F, Gawlitza J, Künstler M, et al. Small airway disease in pulmonary hypertension - additional diagnostic value of multiple breath washout and impulse oscillometry. J Clin Med 2018; 7: E532.

17 Alrumuh A, Gilchrist FJ, Bianco B, et al. Normative values of lung clearance index in children and adults using $\mathrm{SF}_{6}$ as washout gas. J Cyst Fibros 2019; 18: S111.

18 Miller MR, Hankinson J, Brusasco V, et al. Standardisation of spirometry. Eur Respir J 2005; 26: 319-338.

19 Horsley AR, Gustafsson PM, Macleod KA, et al. Lung clearance index is a sensitive, repeatable and practical measure of airways disease in adults with cystic fibrosis. Thorax 2008; 63: 135-140.

20 Singer F, Kieninger E, Abbas C, et al. Practicability of nitrogen multiple-breath washout measurements in a pediatric cystic fibrosis outpatient setting. Pediatr Pulmonol 2013; 48: 739-746.

21 Robinson PD, Latzin P, Verbanck S, et al. Consensus statement for inert gas washout measurement using multiple- and single-breath tests. Eur Respir J 2013; 41: 507-522.

22 Aurora P, Gustafsson P, Bush A, et al. Multiple breath inert gas washout as a measure of ventilation distribution in children with cystic fibrosis. Thorax 2004; 59: 1068-1073.

23 Smith L, Marshall H, Aldag I, et al. Longitudinal assessment of children with mild cystic fibrosis using hyperpolarized gas lung magnetic resonance imaging and lung clearance index. Am J Respir Crit Care Med 2018; 197: 397-400.

24 Davies J, Sheridan H, Bell N, et al. Assessment of clinical response to ivacaftor with lung clearance index in cystic fibrosis patients with a G551D-CFTR mutation and preserved spirometry: a randomised controlled trial. Lancet Respir Med 2013; 1: 630-638.

25 Gustafsson PM, De Jong PA, Tiddens HAW, et al. Multiple-breath inert gas washout and spirometry versus structural lung disease in cystic fibrosis. Thorax 2008; 63: 129-134.

26 Nyilas S, Carlens J, Price T, et al. Multiple breath washout in pediatric patients after lung transplantation. Am J Transplant 2018; 18: 145-153.

27 Walkup LL, Myers K, El-bietar J, et al. ${ }^{129}$ Xe MRI detects ventilation deficits in pediatric stem-cell transplant patients unable to perform spirometry. Eur Respir J 2019; 53: 1801779.

28 Thompson BR, Ellis MJ, Stuart-Andrews C, et al. Early bronchiolitis obliterans syndrome shows an abnormality of perfusion not ventilation in lung transplant recipients. Respir Physiol Neurobiol 2015; 216: 28-34.

29 Dahlstrom H, Murphy JP, Roos A. Cardiogenic oscillations in composition of expired gas. The 'pneumocardiogram'. J Appl Physiol 1954; 7: 335-339.

30 Collier G, Marshall H, Rao M, et al. Observation of cardiogenic flow oscillations in healthy subjects with hyperpolarized ${ }^{3}$ He MRI. J Appl Physiol 2015; 119: 1007-1014. 